\title{
High tuberculosis burden among people living with HIV in southern Mozambique
}

To the Editor:

Tuberculosis (TB) remains an important public health concern, and a leading cause of disease and death worldwide. Mozambique is one of the few high TB burden countries where TB figures have not improved in recent years, with an estimated TB incidence in 2013 of 552 cases per 100000 population [1]. With $58 \%$ of all notified TB cases being HIV-positive, Mozambique also has one of the highest TB/HIV co-infection rates. Published data on the burden of TB or HIV disease in the country are scarce, and improving epidemiological surveillance has been identified as an urgent step to improve TB control [2].

People living with HIV (PLHIV) are at a higher risk of developing active TB, which is the main cause of death among this population, accounting for $26 \%$ of AIDS-related deaths [3, 4]. It has been estimated that in the African region, $31 \%$ of new TB cases in adults were attributable to HIV infection [5]. Most TB incidence measurements among HIV patients come from HIV cohorts [6, 7], clinical trials or mathematical modelling using various strategies described elsewhere [1]. Very few settings, especially in sub-Saharan Africa, provide population-level estimates of TB risk among PLHIV [8, 9]. We determined the incidence rate of TB among HIV-positive and -negative individuals during 2011 in a high HIV burden setting in southern Mozambique.

The study was conducted at the Manhiça Health Research Centre (CISM), located in the rural district of Manhiça, southern Mozambique [10]. This retrospective, population-based epidemiological analysis used three data sources: TB notification data were obtained from the 2011 registries of the National TB Control Program for the District of Manhiça, based on passive surveillance; the population at risk was calculated from the latest official census data (2007) for the District of Manhiça, obtained through the Mozambican National Statistics Institute, and the estimated population growth for 2007-2011, using annual data from CISM's Demographic Surveillance System; and HIV prevalence in the district population was estimated using community-based HIV seroprevalence data from a survey conducted in 2010 [11], which only included adults aged $18-47$ years.

In 2011, laboratory diagnosis of TB was made through conventional smear microscopy using ZiehlNeelsen staining. Culture was only performed in patients who had previously been treated for TB at diagnosis. Samples were processed at CISM's laboratory, which is subject to an External Quality Assurance assessment. TB case definitions followed World Health Organization (WHO) guidelines [1]. TB incidence rate estimates are described with an uncertainty variability range (VR) based on the confidence intervals of the HIV seroprevalence study. In order to avoid bias from false-positive empirical diagnoses, the primary analysis of $\mathrm{TB}$ incidence rate among PLHIV, incidence rate ratio (IRR) calculations and population-attributable fraction (PAF) were based on bacteriologically confirmed TB cases. The PAF to HIV was calculated using the formula $\mathrm{PAF}=[\mathrm{P} \times(\mathrm{IRR}-1)] /[(\mathrm{P} \times \mathrm{IRR}-1)+1)]$, where $\mathrm{P}$ is the prevalence of HIV in the population.

A total of 637 TB cases aged 18-47 years were diagnosed in the district of Manhiça in 2011, of which $53.4 \%$ were male and $278(43.6 \%)$ were confirmed by sputum smear. Bacteriological confirmation was higher among HIV-negative than HIV-positive TB cases (62.2\% versus $39.4 \%$ respectively, $\mathrm{p}<0.001)$. The prevalence of HIV among confirmed TB cases aged 18-47 years was 77.2\% (206 out of 267). Only $15.0 \%$ of patients were reported to be on antiretroviral therapy (ARVs) at TB diagnosis or during TB treatment. Among PLHIV, 14.6\% of confirmed TB cases died during TB treatment, compared with none among HIV-uninfected adults.

The estimated TB incidence rate among adults aged 18-47 years was 456 per 100000 population. The incidence rate of confirmed TB among PLHIV aged 18-47 years was 847 per 100000 (VR 772-941), compared with 168 per 100000 (VR 158-180) among HIV-negative population. Figure 1 shows age and sex-specific TB incidence rates. Among HIV-positive male and female adults, the highest TB incidence rate was observed in those aged 38-47 years, with 1884 and 861 cases per 100000 males and females, respectively.

The IRR of confirmed TB among HIV-infected compared with HIV-uninfected adults was 5.04 (95\% CI 3.77-6.82). The IRR of TB was highest in the 38-47-years age group (fig. 1b). The proportional reduction in TB risk that would be achieved if we eliminated HIV from our adult population (PAF) aged 18-47 years would be $61.7 \%$. 

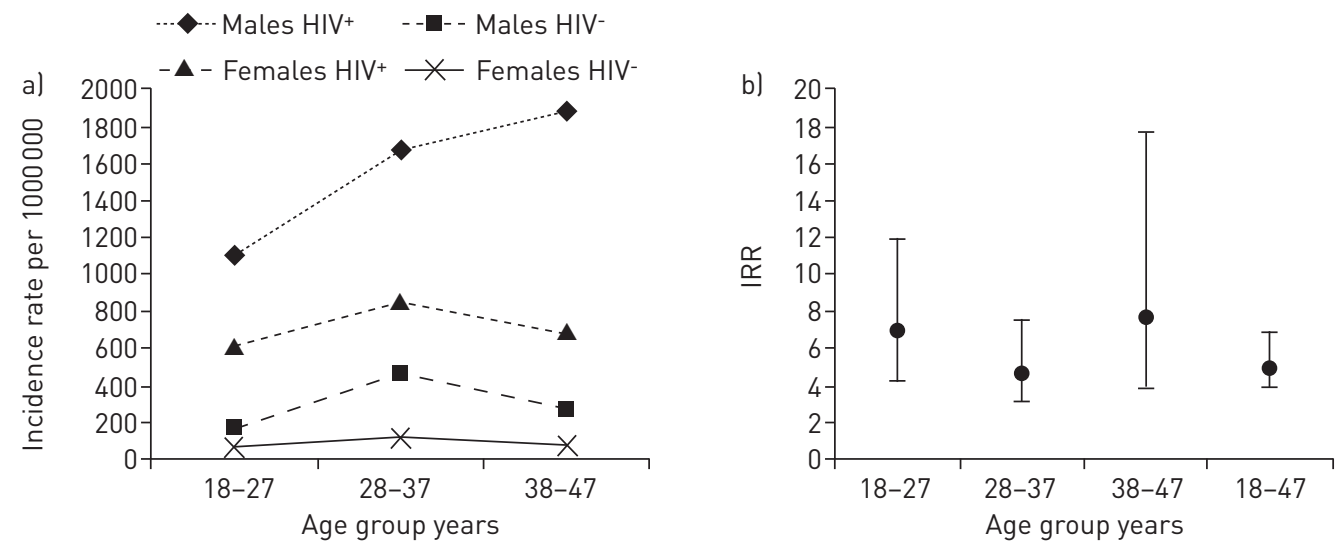

FIGURE 1 a) Incidence rate of confirmed tuberculosis (TB) among the HIV-infected and -uninfected population of the district of Manhiça, Mozambique in 2011. b) Incidence rate ratio (IRR) of TB among the HIV-infected population compared with the HIV-uninfected population by age group in the district of Manhiça in 2011. Whiskers represent $95 \%$ confidence intervals.

To our knowledge, this study is the first study in Mozambique providing estimates of TB incidence among the HIV-infected population and one of the few studies assessing TB incidence among PLHIV in a general population in sub-Saharan Africa [8]. The high TB morbidity and mortality associated with HIV infection found in this analysis underscore the urgent need for improved and integrated control efforts for both diseases [12].

We found an extremely high incidence of laboratory-confirmed TB (847 per 100 000) among HIV-infected adults aged 18-47 years, varying with age and sex, but persistently higher in males. These figures, which depend to a great extent on the country TB prevalence and ARV coverage, are in line with those found in African high-burden countries [6, 8, 13], although some of these studies included active case finding, high-risk populations (e.g. miners) or different age groups. Given the passive nature of TB notification and the presumably low case detection rate for our district (which could be as low as $34 \%$ according to WHO country estimates for Mozambique [1]), the lack of more sensitive diagnostic techniques at that time (e.g. culture or Xpert (Cepheid, Sunnyvale, CA, USA)), and the inherent difficulties in diagnosing TB among PLHIV (often smear negative and presenting with unspecific symptoms), our estimate is a minimum and the true TB incidence among PLHIV is likely to be much higher.

Our findings showing a low IRR of TB among the HIV-infected population (5.04) compared with the HIV-uninfected population are in line with previous published data $[13,14]$, which suggest that the higher the HIV prevalence is in the community, the lower the IRR [3]. However, we are almost certain that the true IRR among HIV-infected versus -uninfected populations was higher due to the higher underreporting of TB among PLHIV. The main explanation for this is the difficulty of diagnosing TB among PLHIV, who more frequently have extrapulmonary/disseminated forms of TB or paucibacillary forms of pulmonary TB, neither of which is detectable through smear examination [15]. The PAF of TB due to HIV (61.5\%) is probably one of the highest ever reported from Africa.

The use of sputum smears as a diagnostic method for TB diagnosis is a serious limitation, as both culture and newer molecular methods have higher sensitivity and specificity, especially in PLHIV. Moreover, taking into account that almost $50 \%$ of the population of the country is younger than 15 years, we cannot extend our conclusions to this important age group, nor could we assess the impact of other potential factors that could help to stratify the risk among HIV infected population, such as ARV coverage, the level of immunosuppression or the time from HIV infection to TB diagnosis. The low number of TB patients on ARV needs further evaluation to assess whether these data reflects poor ARV coverage or poor reporting due to the absence of integrated TB/HIV management at that time.

In conclusion, this study shows an enormous burden of $\mathrm{TB}$, in terms of incident cases and case fatality, among PLHIV in a rural district of Southern Mozambique. Acknowledging there is a high underdiagnosis, the true burden of TB among PLHIV could be enormous. These findings call for urgent public health interventions to reverse current TB/HIV epidemic. 
Alberto L. García-Basteiro, ${ }^{1,2}$, Elisa López-Varela ${ }^{1,2}$, Durval Respeito ${ }^{1}$, Raquel González ${ }^{2}$, Denise Naniche ${ }^{2}$, Ivan Manhiça ${ }^{3}$, Eusebio Macete ${ }^{1}$, Frank Cobelens ${ }^{4}$ and Pedro L. Alonso ${ }^{1,2}$

${ }^{1}$ Manhiça Health Research Center (CISM), Maputo, Mozambique. ${ }^{2}$ ISGlobal, Barcelona Centre for International Health Research (CRESIB), Hospital Clínic - Universitat de Barcelona, Barcelona, Spain. ${ }^{3}$ Ministry of Health, National Tuberculosis Program, Maputo, Mozambique. ${ }^{4}$ Amsterdam Institute for Global Health and Development, Academic Medical Centre, Amsterdam, The Netherlands.

Correspondence: Alberto L. García-Basteiro, Manhiça Health Research Center, Vila da Manhiça, CP 1929 Maputo, Mozambique. E-mail: alberto.garcia-basteiro@manhica.net

Received: Aug 082014 | Accepted after revision: Oct 132014 | First published online: Nov 132014

Conflict of interest: None declared.

Acknowledgements: We thank Sergi Sanz, Elisa de Lazzari (both ISGlobal, CRESIB, Hospital Clínic - Universitat de Barcelona, Barcelona, Spain), Alberto Bila Junior and Elisa Fernández Cooke (both CISM, Maputo, Mozambique) for their support with the statistical analysis, data management and comments on the manuscript.

\section{References}

1 World Health Organization. Global Tuberculosis Report 2013. WHO/HTM/TB/2013.11. Geneva, WHO, 2013.

2 García-Basteiro AL, López-Varela E, Manhiça I, et al. Mozambique faces challenges in the fight against tuberculosis. Lancet 2014; 383: 215-216.

3 Getahun H, Gunneberg C, Granich R, et al. HIV infection-associated tuberculosis: the epidemiology and the response. Clin Infect Dis 2010; 50: Suppl. 3, S201-S207.

4 Podlekareva DN, Panteleev AM, Grint D, et al. Short- and long-term mortality and causes of death in HIV/ tuberculosis patients in Europe. Eur Respir J 2014; 43: 166-177.

5 Corbett EL, Bandason T, Cheung YB, et al. Epidemiology of tuberculosis in a high HIV prevalence population provided with enhanced diagnosis of symptomatic disease. PLoS Med 2007; 4: e22.

6 Addis Alene K, Nega A, Wasie Taye B. Incidence and predictors of tuberculosis among adult people living with human immunodeficiency virus at the University of Gondar Referral Hospital, Northwest Ethiopia. BMC Infect Dis 2013; 13: 292.

7 Lawn S, Myer L, Edwards D, et al. Short-term and long-term risk of tuberculosis associated with CD4 cell recovery during antiretroviral therapy in South Africa. AIDS 2013; 23: 1717-1725.

8 Yuen CM, Weyenga HO, Kim A, et al. Comparison of trends in tuberculosis incidence among adults living with HIV and adults without HIV - Kenya, 1998-2012. PLoS One 2014; 9: e99880.

9 Gupta A, Wood R, Kaplan R, et al. Tuberculosis incidence rates during 8 years of follow-up of an antiretroviral treatment cohort in South Africa: comparison with rates in the community. PLoS One 2012; 7: e34156.

10 Sacoor C, Nhacolo A, Nhalungo D, et al. Profile: Manhica Health Research Centre (Manhica HDSS). Int J Epidemiol 2013; 42: 1309-1318.

11 González R, Munguambe K, Aponte J, et al. High HIV prevalence in a southern semi-rural area of Mozambique: a community-based survey. HIV Med 2012; 13: 581-588.

12 Sester M, Giehl C, McNerney R, et al. Challenges and perspectives for improved management of HIV/ Mycobacterium tuberculosis co-infection. Eur Respir J 2010; 36: 1242-1247.

13 Corbett EL, Charalambous S, Moloi VM, et al. Human immunodeficiency virus and the prevalence of undiagnosed tuberculosis in African gold miners. Am J Respir Crit Care Med 2004; 170: 673-679.

14 Glynn JR. Resurgence of tuberculosis and the impact of HIV infection. Br Med Bull 1998; 54: 579-593.

15 Schutz C, Meintjes G, Almajid F, et al. Clinical management of tuberculosis and HIV-1 co-infection. Eur Respir J 2010; 36: 1460-1481.

\section{How is Xpert MTB/RIF being implemented in 22 high tuberculosis burden countries?}

To the Editor:

Accurate and rapid diagnosis is crucial for tuberculosis control by ensuring a timely start to treatment and reducing transmission. In 2012, almost one third of tuberculosis cases were not diagnosed and/or reported to national tuberculosis programmes (NTPs), and $<25 \%$ of estimated incident multidrug-resistant (MDR) cases were diagnosed [1]. Xpert MTB/RIF (Cepheid, Sunnyvale, CA, USA), a nucleic acid amplification test, was recommended in 2010 by the World Health Organization (WHO) for detection of HIV-associated 